Pacific Journal of Mathematics

A NEW MEASURE OF A PARTIAL DIFFERENTIAL FIELD 


\title{
A NEW MEASURE OF A PARTIAL DIFFERENTIAL FIELD EXTENSION
}

\author{
ISRAEL ZUCKERMAN
}

\begin{abstract}
Let $G$ be a differential field of characteristic zero with the commuting derivations $d_{1}, \cdots, d_{m}$. If $F$ is a differential subfield of $G$, the algebraic and differential degrees of transcendence of $G$ over $F$, denoted respectively by $d(G / F)$ and $d . d(G / F)$ are numerical invariants of the extension. Unlike the ordinary differential case $(m=1) d . d .(G / F)=0$ does not imply that $d(G / F)$ is finite. In this paper an intermediate measure of the extension is constructed, called the limit vector. The first and last components of this vector correspond to $d . d(G / F)$ and $d(G / F)$ respectively, and the limit vector is additive.
\end{abstract}

Similar concepts have been developed independently by Kolchin in a work not yet published.

Characteristic sets of prime ideals as defined in [6] play a prominent role in the development of the limit vector, as well as in the development of other results of this paper which do not depend on the limit vector. Further, it is shown that an intermediate field of a finitely generated extension is finitely generated. Kolchin has a prior, but different proof of this. Kolchin's analog of Lüroth's Theorem [2,3] is extended and some results on characteristic sets of length one are obtained.

Raudenbush [5] shows that the dependence axioms of Van der Waerden [7] are satisfied by differential dependence. It is indicated below that these axioms are more readily established by use of the limit vector. A further result is that a proper specialization of $F[a]$ must reduce the limit vector only if $a$ has a characteristic set of length one over $F$. A short proof of a theorem of Delsarte [1] on partial linear homogeneous differential equations concludes the paper.

2. Ordering the derivatives. The main source of this subject is Ritt [6], especially $\S 8$ and $\S 2$ of Chapter VIII and $\S \S 1-16$ of Chapter IX. In general, the terminology and notation are as in [6]. Consider the differential ring $F\left\{y_{1}, \cdots, y_{r}\right\}$, where the $y_{i}$ are differential indeterminates. Then $D=d_{1}^{i_{1}} \cdots d_{m}^{i_{m}}$ will denote a derivative, i.e., the composite of derivations. We associate with $D$ the vector $\left(i_{1}, \cdots, i_{m}\right)$. The sum of the $i_{k}$ is called the order of $D$, or of the associated vector,

Received January 23, 1964. This paper is the author's doctoral dissertation submitted to Rutgers University in March 1963 under the direction of Professor Richard Cohn. The work was partly supported by the National Science Foundation Grant G-19636. 
or of $D y_{j} . y_{j}$ is to be considered a derivative of itself of zero order. Let $w$ denote an arbitrary $y$-derivative. A set of marks will be assigned to the $d_{i}$ and $y_{i}$ to achieve a complete ordering of the $w$. In doing so, the primary objective will be to have $w_{1}$ precede $w_{2}$ if the order of $w_{1}$ is less than the order of $w_{2}$. Associate with each $d_{i}$, the marks $u_{i 1}, \cdots, u_{i, m+2}$; where $u_{i 1}=1, u_{i 2}=0$, and for $k>2: u_{i k}=0$ for $k \neq i+2$, $u_{i k}=1$ for $k=i+2$. Associate with each $y_{i}$ the marks $v_{i 1}, \cdots, v_{i, m+2}$, where $v_{i 2}=i$ and $v_{i k}=0$ for $k \neq 2$. Let $w=d_{1}^{i_{1}} \cdots d_{m}^{i_{m}} y_{i}$. Then $w$ is assigned $m+2$ marks as follows: The $j$ th mark of $w$ is $v_{i j}+i_{1} u_{1 j}+$ $\cdots+i_{m} u_{m j}$. Let $w_{1}$ and $w_{2}$ be two $y$-derivatives with marks $a_{i}$ and $b_{i}$ respectively. Then we shall say that $w_{1}$ precedes $w_{2}$ or succeeds $w_{2}$ according as the first nonzero difference $b_{i}-a_{i}$ is positive or is negative. As one can easily verify the system of marks introduced here achieves the desired complete ordering of the $y$-derivatives. Such ordering will prevail throughout, with the exception of $\S 12$.

3. A transcendence basis for $a_{1}, \cdots, a_{r}$ over $\mathrm{F}$. Let $a_{1}, \cdots, a_{r}$ be elements in $G$. If the $a_{i}$ are differentially dependent over $F$, let $P$ be the prime ideal in $F\left\{y_{1}, \cdots, y_{r}\right\}$ with $\left(a_{1}, \cdots, a_{r}\right)$ as a generic zero, and let $C=\left(A_{1}, \cdots, A_{k}\right)$ be a characteristic set of $P . \quad(C$ is said to be a characteristic set of the $a_{i}$ over $F$.) Denote the leader and separant of $A_{i}$ by $p_{i}$ and $S_{i}$ respectively. If $w$ is a derivative of some $p_{i}$, it will be called principal; otherwise, it will be called parametric. We will call an $a$-derivative principal if it admits a representation $w(a)$ where $w$ is principal; otherwise, it will be called parametric. If the $a_{i}$ are differentially independent over $F$, all $a$-derivatives will be considered to be parametric. The ordering of the $y$-derivatives carries over to the ordering of the symbols for the $a$-derivatives.

THEOREM 1. The parametric derivatives of $a_{1}, \cdots, a_{r}$ are distinct and constitute a transcendence basis for $F\left(a_{1}, \cdots, a_{r}\right)$ over $F$. Moreover, a principal a-derivative depends algebraically on parametric derivatives of equal or lower order.

Proof. If the $a_{i}$ are differentially independent over $F$, the proof is immediate. Now assume that the $a_{i}$ are differentially dependent over $F$, with the associated prime ideal $P$ and the characteristic set $C$. Since $C$ is a characteristic set of $P$, every differential polynomial, (d.p.), in $P$ involves a derivative of some $p_{i}$. Hence the parametric $a$-derivatives are distinct and algebraically independent over $F$. This proves the first statement of the theorem.

Now, the principal $a$-derivatives are completely ordered with $p_{1}(a)$ as the first derivative. Since $p_{1}$ is the leader of $A_{1}, p_{1}(a)$ depends solely on parametric derivatives. Let $w\left(a_{j}\right)$ be a principal $a$-derivative suc- 
ceeding $p_{1}(a)$. If $w$ is a proper derivative of some leader, say $p_{i}$, then an appropriate derivative $D$ of $A_{i}$ yields

$0=D A_{i}\left(a_{1}, \cdots, a_{r}\right)=S_{i}\left(a_{1}, \cdots, a_{r}\right) w\left(a_{j}\right)+B\left(a_{1}, \cdots, a_{r}\right)$, where the $a$-derivatives in $S_{i}(\alpha)$ and $B(\alpha)$ precede $w\left(\alpha_{j}\right)$. $w$ may also be some $p_{i}$ for $i>1$. In either case, $w\left(\alpha_{j}\right)$ depends on principal and parametric derivatives whose symbols precede the symbol for $w\left(a_{j}\right)$. By induction, each of these principal derivatives depends on preceding parametric derivatives; hence on parametric derivatives which precede $w\left(a_{j}\right)$. Thus, $w\left(a_{j}\right)$ depends on preceding parametric derivatives, concluding the proof.

4. Restriction to $\alpha$-derivatives with orders not exceeding $n$. Let $A$ be a subset of $G$, and $n$ a positive integer. Then the set of derivatives of elements of $A$ of order not exceeding $n$ will be denoted by $(n ; A)$. Let $F^{\prime}$ be an ordinary field contained in $G$. Then, as is customary, $F^{\prime}(n ; A)$ will denote the ordinary field extension obtained by adjoining $(n ; A)$ to $F^{\prime} . d\left(F^{\prime}(n ; A) / F^{\prime}\right)$ will be denoted by $h\left(F^{\prime}, n ; A\right)$ or simply by $h(n)$ if no ambiguity arises. (This type of abbreviation is repeated throughout.)

With this notation the following corollary is immediate, noting that the ordering of the $a$-derivatives is such that a principal derivative depends on parametric derivatives of equal or lower order.

COROLLARY 1. $h\left(F, n ; \alpha_{1}, \cdots, a_{r}\right)$ is the number of parametric derivatives of the $a_{i}$ of order not exceeding $n$.

THEOREM 2. For $n$ sufficiently large,

$$
h\left(F, n ; a_{1}, \cdots, a_{r}\right)=H\left(F, n ; a_{1}, \cdots, a_{r}\right) / m !,
$$

where $H(n)$ is a polynomial in $n$ with integral coefficients. $H(n)=0$, or has degree $t \leqq m$ and leading coefficient $c_{t}>0$.

Proof. Let $p(n)$ denote the number of derivatives of some $y$ of order not exceeding $n$. Then

$$
p(n)=C(n+m, m)=(n+m)(n+m-1) \cdots(n+1) / m !
$$

Hence, if the $\alpha_{i}$ are differentially independent over $F, h(n)=r p(n)$ and the theorem is true in such case with $t=m$ and $c_{t}=r$.

Now assume that the $a_{i}$ are differentially dependent over $F$ with leaders $p_{i}$ of a characteristic set for the $\alpha_{i}$ over $F$. Partition the $p_{i}$ into subsets $R_{j}$, each consisting of derivatives of the same $y_{j}$. Let $q_{1}, \cdots, q_{s}$ be one such subset. For each nonempty subset $T$ of $\left\{q_{1}, \cdots, q_{s}\right\}$, let $u(T)$ be the $m$-vector with $k$ th component, $k(T)$, equal to the 
maximum of the $k$ th components of the vectors associated with the $q_{i}$ in $T$, and let $v_{T}$ be the sum of the $k(T)$. Let $n$ be a positive integer greater than the maximum of the $v$ over all $R_{j}$. Then an $m$-vector will be called a multiple of $u(T)$ if each of its components is not less than the corresponding component of $u(T)$, and if the sum of its components does not exceed $n$. Let $S(T)$ denote the set of multiples $u(T)$, and let $N(T)$ denote the number of elements in $S(T)$. Let $B(T)$ denote the number of elements in $T$.

Then,

$$
N(T)=C\left(n+m-v_{T}, m\right) .
$$

Note that $p(n)$ is of the form (1) with $v=0$. Therefore we extend the set of subsets $T$ to include the empty set $\varphi$ and define $v_{\varphi}=0$, $N(\varphi)=p(n)$. The number of parametric derivatives of $y_{j}$ of order not exceeding $n$ is obtained by subtracting from $p(n)$ the number of elements in the union of the $S(T)$. Thus, it is equal to

$$
\sum_{T}(-1)^{B(T)} N(T)
$$

(2) is the sum of $2^{3}$ expressions, each of the form (1). The term of highest degree in $n$ in each expression is the same, $n^{m} / m$ ! Since there are as many positive as negative expressions in (2), the sum of the terms in $n^{m}$ is zero. Furthermore, by consideration of large $n$ we see that the effective leading coefficient of (2) is positive.

The sum of the number of parametric derivatives of the $R_{j}$ is then a polynomial with the desired properties. (An empty $R_{j}$ contributes $p(n)$ elements.)

The following corollary is immediate.

CoRollary 1. Let $s$ be an integer with $0 \leqq s \leqq m$. If $a_{1}, \cdots, a_{r}$ are differentially dependent over $F$, then

$$
\lim _{n \rightarrow \infty} n^{s} h(n) / p(n)= \begin{cases}0, & \text { if } s<m-t ; \\ c_{t}, & \text { if } s=m-t ; \\ \infty, & \text { if } s>m-t .\end{cases}
$$

If $a_{1}, \cdots, a_{r}$ are differentially independent over $F$, then

$$
\lim _{n \rightarrow \infty} n^{8} h(n) / p(n)= \begin{cases}r, & \text { for } s=0 ; \\ \infty, & \text { for } 0<s<m .\end{cases}
$$

5. Introduction of the limit vector. We may now define

$$
L_{s}\left(F, a_{1}, \cdots, a_{r}\right)=\lim _{n \rightarrow \infty} n^{s} h(n) / p(n) \quad(s=0, \cdots, m),
$$


and

$$
L\left(F, a_{1}, \cdots, a_{r}\right)=\left(L_{0}, \cdots, L_{m}\right) .
$$

The latter will be called the limit vector of $a_{1}, \cdots, a_{r}$ over $F$.

The following remarks are evident.

$$
d . d\left(F\left\langle a_{1}, \cdots, a_{r}\right\rangle / F\right)=r \Leftrightarrow L_{0}=r .
$$

(2) For a single element $a, d . d(F\langle a\rangle / F)=0 \Leftrightarrow L_{0}=0$.

We will later show that $d . d\left(F\left\langle a_{1}, \cdots, a_{r}\right\rangle / F\right)=L_{0}$, subsuming (1) and (2).

$$
d\left(F\left\langle a_{1}, \cdots, a_{r}\right\rangle \mid F\right)=k<\infty \Leftrightarrow L_{m}=k m !
$$

In particular, if each $a_{i}$ is algebraic over $F, L_{s}=0$ for all $s$.

6. Results on $L_{1}$. The following corollaries follow from the proof of Theorem 2. In this section, $a$ will be differentially algebraic over $F$ with characteristic set $C=A_{1}, \cdots, A_{k}, 0<k<\infty . W_{i}$ will denote the vector associated with the leader of $A_{i}$.

COROLlary 2.

$$
L_{1}(F ; a)=m \sum_{T}(-1)^{B(T)+1} v_{T} .
$$

In particular, this shows that $L_{1}(F ; a)$ is divisible by $\mathrm{m}$.

Proof. The coefficient of $n^{m-1}$ in $m ! C\left(n+m-v_{T}, m\right)$ is

$$
\left(m-v_{T}\right)+\cdots+\left(1-v_{T}\right)=m(m+1) / 2-m v_{T} .
$$

The first term in (3) is the same for each $v_{T}$. Since there are as many positive as negative expressions in (2), these terms cancel in computing $C_{m-1}$, and the desired result follows.

As a special case of Corollary 2, we have the following.

COROLlaRY 3. If a has a characteristic set of length one over $F$, then $L_{1}(F ; a)=m g$ where $g$ is the order of such characteristic set.

The result of Corollary 2 may be carried further so as to depend more directly on the leaders of the characteristic set. We need the following lemma.

LEMma. Let $u(1), \cdots, u(k), 1 \leqq k<\infty$, be a sequence $S$ of real numbers with $u(1)=\min (u(i))$. For each subsequence $T$ of $S$, let $B(T)$ and $M(T)$ denote respectively the number and maximum of its elements. Then, 


$$
\sum_{T}(-1)^{B(T)+1} M(T)=u(1)
$$

Proof. Let $T^{\prime}$ denote a $T$ which does not have $u(1)$ as its first Felement; and let $T^{\prime \prime}$ be the sequence obtained by adjoining $u(1)$ at the beginning of $T^{\prime}$. Then, with the exception of $T=u(1)$, the $T$ can be partitioned into pairs of $T^{\prime}$ and $T^{\prime \prime}$ such that

$$
(-1)^{B\left(T^{\prime}\right)+1} M\left(T^{\prime}\right)+(-1)^{B\left(T^{\prime \prime \prime}\right)+1} M\left(T^{\prime \prime}\right)=0 .
$$

Thus, the desired result is obtained.

CoRollary 4 . Let $w_{j}$ denote the minimum of the jth components of the $W_{i}$. Then

$$
L_{1}(F ; a)=m \sum_{j=1}^{m} w_{j} \cdot
$$

Proof. By the lemma, the sum of the $j$ th components of the vectors associated with the $T$, with the appropriate signs affixed, is $w_{j}$.

Hence, if we sum the $(-1)^{B(T)+1} v_{T}$ component-wise, the result of Corollary 2 yields $L_{1}(F ; a)=m \sum_{j=1}^{m} w_{j}$, as desired.

Note that Corollary 4 implies that $L_{1}(F ; a)=0$ if and only if $w_{j}=$ 0 for all $j$.

Corollary 5. Let $A_{t}$ of order $v$ belong to $C$, and let $k>1$. Then $L_{1}(F ; a)<m v$.

Proof. If for $j=1, \cdots, m$, the $j$ th component of $W_{t}$ is the minimum of the $j$ th components of the $W_{i}$, then for $i \neq t, A_{i}$ would not be reduced with respect to $A_{t}$.

We are now in a position to prove a converse of Corollary 3.

CoRollary 6. If $L_{1}(F ; a)=m g, 0 \leqq g<\infty$, and if a satisfies an irreducible d.p. A over $F$ of order $g$, then $A$ is a characteristic set for a over $F$.

Proof. Since $a$ satisfies $A$, there exists a $d . p . A^{\prime}$ in $C$ with order $g^{\prime} \leqq g$. By Corollary 5 , if $k>1, L_{1}(F ; a)<m g^{\prime} \leqq m g$. Hence, $C=A^{\prime}$. By Corollary $3, g^{\prime}=g$ and $A^{\prime}$ has the same order as $A$. Since $A$ has zero remainder with respect to $A^{\prime}$ and is irreducible, $A=c A^{\prime}$ where $c$ is in $F$; hence, $A$ is a characteristic set for $a$ over $F$.

\section{7, Additivity of the limit vector.}

Lemma 1. Let $A$ be a finite set of elements contained in an ex- 
tension of $F$. If $k, q$, and $r$ are nonnegative integers and $s$ an integer such that $0 \leqq s \leqq m$, then

$$
\lim _{n \rightarrow \infty}\left[(n+k)^{s} h(n+q, A) / p(n+r)\right]=\lim _{n \rightarrow \infty}\left[n^{s} h(n, A) / p(n)\right] .
$$

Proof.

$$
\frac{(n+k)^{s} h(n+q, A)}{p(n+r)}=\frac{(n+q)^{s} h(n+q, A)}{p(n+q)} \frac{(n+k)^{s}}{(n+q)^{s}} \frac{p(n+q)}{p(n+r)} .
$$

Clearly,

$$
\lim _{n \rightarrow \infty} \frac{p(n+q)}{p(n+r)}=1, \quad \text { and } \quad \lim _{n \rightarrow \infty} \frac{(n+k)^{s}}{(n+q)^{s}}=1
$$

Furthermore,

$$
\lim _{n \rightarrow \infty} \frac{(n+q)^{s} h(n+q, A)}{p(n+q)}=\lim _{n \rightarrow \infty} \frac{n^{s} h(n, A)}{p(n)}
$$

LEMMA 2. Let $A$ be a set and $b$ an element contained in an extension of $F$ with b differentially algebraic over $F\langle A\rangle$. Let $t$ be the maximal order of derivatives of $A$ appearing in a characteristic set $C$ for $b$ over $F\langle A\rangle$. Let $A^{\prime}$ be a set of A-derivatives containing $(n+t ; A)$. Then,

$$
h(F\langle A\rangle, n ; b)=h\left(F\left(A^{\prime}\right), n ; b\right) .
$$

Proof. Let $S(n)$ denote the set of parametric derivatives of $b$ of order not exceeding $n$ with respect to $C$. Then $S(n)$ is algebraically independent over $F\left(A^{\prime}\right)$. Furthermore, $S(n)$ is an algebraic spanning set for $(n ; b)$ over $F\left(A^{\prime}\right)$ since in the proof of Theorem 1 only derivatives with orders not exceeding $n$ of the d.p. in $C$ are present in the algebraic relations obtained for the principal derivatives.

Note that the result holds more readily if $b$ is differentially transcendental over $F\langle A\rangle$.

\section{THEOREM 3.}

$$
L\left(F ; a_{1}, \cdots, a_{r}\right)=\sum_{i=1}^{r} L\left(F\left\langle a_{1}, \cdots, a_{i-1}\right\rangle ; a_{i}\right) .
$$

Proof. For $i=1, \cdots, r-1$, if $a_{i+1}$ is differentially transcendental over $F\left\langle a_{1}, \cdots, a_{i}\right\rangle$, let $t_{i}=0$. Otherwise, $t_{i}$ be the maximal order of derivatives of $a_{1}, \cdots, a_{i}$ in a characteristic set for $a_{i+1}$ over $F\left\langle a_{1}, \cdots, a_{i}\right\rangle$. Then, 


$$
\begin{aligned}
F(n+ & \left.t_{1}+\cdots+t_{r-1} ; a_{1}, \cdots, a_{r}\right) \\
& \supset F\left(n+t_{1}+\cdots+t_{r-1} ; a_{1}\right)\left(n+t_{2}+\cdots+t_{r-1} ; a_{2}\right) \\
& \cdots\left(n+t_{r-1} ; a_{r-1}\right)\left(n ; a_{r}\right) \supset F\left(n ; a_{1}, \cdots, a_{r}\right) .
\end{aligned}
$$

Then, by additivity of the transcendence degree and Lemma 2,

$$
\begin{aligned}
& h\left(F, n+t_{1}+\cdots+t_{r-1} ; a_{1}, \cdots, a_{r}\right) \\
& \quad \geqq \sum_{i=1}^{r} h\left(F\left\langle a_{1}, \cdots, a_{i-1}\right\rangle, n+t_{i}+\cdots+t_{r-1} ; a_{i}\right) \\
& \quad \geqq h\left(F, n ; a_{1}, \cdots, a_{r}\right) .
\end{aligned}
$$

Multiplying by $n^{s} / p(n)$, then taking the limit of the resulting expressions as $n$ approaches $\infty$, by application of Lemma 1 , the desired result is obtained.

8. Extension of the limit vector to a measure of an arbitrary differential field extension. Let $f(G)$ denote the set of finite subsets of an extension $G$ of $F$, including the null set. For $S$ and $T$ in $f$, suppose that $k$ is the first component for which $L(F ; S)$ and $L(F ; T)$ differ, and that $L_{k}(F ; S)<L_{k}(F ; T)$. Then we will write $L(F ; S)<L(F ; T)$ and we may define $L(G / F)=\sup _{s \in s} L(F ; S)$.

COROLLARY 1. If $G$ is finitely generated over $F$, i.e., $G=F\left\langle a_{1}, \cdots, a_{r}\right\rangle$, then $L(G / F)=L\left(F ; a_{1}, \cdots, a_{r}\right)$.

Proof. Let $A=\left\{a_{1}, \cdots, a_{r}\right\}$ and $B$ belong to $f$. Then $G=F\langle A, B\rangle$. By Theorem $3, L(F ; B) \leqq L(F ; A, B)=L(F ; A)+L(F\langle A\rangle, B)$. Since $B \subset F\langle A\rangle, L(F\langle A\rangle ; B)=0$. Hence, $L(F ; B) \leqq L(F ; A)$ for all $B$ in $f$. Immediate consequences are:

CoRollary 2. Given $G=F\langle A\rangle=F\langle B\rangle$, where both $A$ and $B$ belong to $f$. Then $L(F ; A)=L(F ; B)$.

CoRollary 3. If $H$ is finitely generated over $G$, and $G$ is finitely generated over $F$, then

$$
L(H / F)=L(H / G)+L(G / F) .
$$

9. A general additivity theorem.

TheOREM 4. Given $F \subset G \subset H$. Then, $L(H / F)=L(G / F)+L(H / G)$.

Proof. (Part I): To prove $L(H / F) \geqq L(G / F)+L(H / G)$. Let $g(H)$ denote the set of finite subsets of $H$, including the null set, which contain no elements of $G$. Then every $V$ in $f(H)$ is the unique union 
of an $S$ in $f(G)$ and a $T$ in $g(H)$, and conversely. For a particular $V=$ $S \cup T$

$$
L(F ; V)=L(F ; S)+L(F\langle S\rangle ; T \geqq L(F ; S)+L(G ; T) .
$$

Hence,

$$
\begin{aligned}
\sup _{\nabla \in f(H)} L(F ; V) \geqq \sup _{V}(L(F ; S)+L(G ; T)) \\
\quad \sup _{S \in F(G)} L(F ; S)+\sup _{T \in g(H)} L(G ; T)=L(G ; F)+L(H ; G) .
\end{aligned}
$$

Proof. (Part II): To prove $L(H / F) \leqq L(G / F)+L(H / G)$. It suffices to show that $L\left(F\left\langle h_{1}, \cdots, h_{r}\right\rangle / F\right) \leqq L(H / G)+L(G / F)$ for any finite subset $h_{1}, \cdots, h_{r}$ of $H$. Let $H_{1}=G\left\langle h_{1}, \cdots, h_{r}\right\rangle$, and let $P$ be the prime ideal in $G\left\{y_{1}, \cdots, y_{r}\right\}$ with $h_{1}, \cdots, h_{r}$ as a generic zero. If $P=0$, let $G_{1}=F$. Otherwise, let $G_{1}=F\langle A\rangle$, where $A$ is the set of coefficients of a characteristic set $C$ of $P$. Let $P_{1}$ be the prime ideal in $G_{1}\left\{y_{1}, \cdots, y_{r}\right\}$ with $h_{1}, \cdots, h_{r}$ as a generic zero. Then $C$ is also a characteristic set of $P_{1}$. Hence, $L\left(H_{1} / G\right)=L\left(G_{1}\left\langle h_{1}, \cdots, h_{r}\right\rangle / G_{1}\right)$.

By additivity for the finitely generated case,

$$
\begin{aligned}
L\left(F\left\langle h_{1}, \cdots, h_{r}\right\rangle / F\right. & \leqq L\left(G_{1}\left\langle h_{1}, \cdots, h_{r}\right\rangle / F\right) \\
& =L\left(G_{1}\left\langle h_{1}, \cdots, h_{r}\right\rangle / G_{1}\right)+L\left(G_{1} / F\right) \\
& =L\left(H_{1} / G\right)+L\left(G_{1} / F\right) \\
& \leqq L(H / G)+L(G / F) .
\end{aligned}
$$

10. Remarks on $L_{0}$. (1) Almost all of the previous results on $L_{0}$ could be obtained more readily: If $a$ is differentially algebraic over $F$, it is sufficient to consider a single d.p. $B(y)$ in $F\{y\}$ which has $a$ as a nonsingular solution. (i.e., $a$ does not annul the separant of $B(y)$.)

(2) In order to establish the theory of differential dependence for arbitrary $m$, Raudenbush showed in [3] that differential dependence satisfied the dependence axioms of Van der Waerden [5]. However this theory follows immediately from the results on $L_{0}$. Furthermore, since it suffices to consider differentially independent subsets of $G$ in determining $L_{0}(G / F)$, it is clear that d.d $(G / F)=L_{0}(G / F)$. Hence the additivity of d.d. follows from the additivity of $L_{0}$.

\section{A result on finitely generated extensions.}

THEOREM 5. Given $F \subset G \subset H=F\left\langle a_{1}, \cdots, a_{r}\right\rangle$. Then $G$ is finitely generated over $F$.

Proof. If the $a_{i}$ are differentially independent over $G$, then it follows readily that $F=G$. Hence the theorem is true in such case.

If the $a_{i}$ are differentially dependent over $G$ let $Q$ be the set of 
coefficients of a characteristic set $A_{1}, \cdots, A_{k}$ of the $a_{i}$ over $G$. Then we assert that $G=F\langle Q\rangle$. For let $p_{i}$ be the leader of $A_{i}$ and $R$ the set of parametric derivatives of the $a_{i}$. Construct

$$
G^{\prime}=F\langle Q\rangle, \quad G^{*}=G^{\prime}(R), \quad G^{*}=G(R) .
$$

Then

$$
H=G^{* *}\left(p_{1}, \cdots, p_{k}\right)=G^{*}\left(p_{1}, \cdots, p_{k}\right)
$$

and

$$
\left[H: G^{*}\right]=\left[H: G^{*}\right]=\prod_{i=1}^{k}\left(\operatorname{deg} A_{i} \text { in } p_{i}\right)<\infty \text {. }
$$

Thus

$$
G^{*}=G^{*} \text {. }
$$

Moreover, since the parametric derivatives of the $a_{i}$ are transcendental over both $G$ and $G^{\prime},\left[G: G^{\prime}\right]=\left[G^{*}: G^{*}\right]=1$. Hence, $G=G^{\prime}$ and the theorem is proved.

12. Characteristic sets of length one and Lüroth's theorem. Kolchin [1] and [2] proves the differential analog of Lüroth's theorem for ordinary differential fields of characteristic zero; explicitly, if $F \subset G \subset F\langle y\rangle$, then $G=F\langle a\rangle$, for some $a$ in $G$. With minor changes Kolchin's proof goes through for the partial case, provided that $y$ has a characteristic set of length one over $G .{ }^{1}$

Using the ideas in [1] and [2], we establish the following converse.

Theorem 6. If $F \subset F\langle a\rangle \subset F\langle y\rangle$, where $a \notin F$, then $y$ has $a$ characteristic set of length one over $F\langle a\rangle$.

Proof. Since $a \in F\langle y\rangle, a=P(y) / Q(y)$, where $P$ and $Q$ may be taken to be relatively prime d.p. over $F$. We assert that $A(z)=$ $a Q(z)-P(z)$ is a characteristic set of $y$ over $F\langle a\rangle$. To begin with, $A$ is clearly irreducible over $F\langle a\rangle$.

Let $Y$ be the prime ideal over $F\langle a\rangle$ with $y$ as a generic zero. Let $X$ be the prime ideal over $F\langle a\rangle$ with $A$ as a characteristic set, and let $x$ be a generic zero of $X . \quad x$ is differentially transcendental over $F$; for otherwise $a$, and hence $y$ would be differentially algebraic over $F$. Therefore, mapping $y$ onto $x$ determines an $F$-isomorphism

1. In Kolchin's proof, [2] on the 9 th and 10th lines from the top on page 400 , the result follows by considering the derivatives ordered so that every $z$ derivative is higher than every $y$ derivative. On the 16 th line from the top on the same page, the $y$ derivatives need to be considered higher. These orderings differ from those used in this paper. 
of $F\langle y\rangle$ onto $F\langle x\rangle$. Under this isomorphism, a remains fixed, for $a=P(y) / Q(y)=P(x) / Q(x)$. Hence the isormorphism leaves $F\langle a\rangle$ fixed. Thus $y$ and $x$ satisfy the same d.p.'s over $F\langle a\rangle$, and $y$ is a generic zero of $Q$. Hence, $X=Y$ and $A$ is a characteristic set of $X$.

13. The length of a characteristic set is not a property of the extension. If $F\langle a\rangle=F\langle b\rangle$, and if $a$ has a characteristic set of length one over $F$, must this also be true of $b$ ? The answer is in the negative as the following example will indicate.

ExAMPLe. Let $u$ denote a differential indeterminate and let $m=2$ with the derivations denoted by subscripts $x$ and $y$ as in $u_{x}$ and $u_{y}$.

Let $P$ be the set of d.p. with zero remainder with respect to $u_{x}$. Then $P$ is a prime differential ideal. Since the initial and separant of $u_{x}$ are both one, $P$ is also generated by $u_{x}$ over $F$. Let $a$ be a generic zero of $P$ 。 Define

$$
b=a+p a_{y},
$$

where $p$ is in $F$ and is differentially transcendental over the field of rationals contained in $F$. Then

$$
\begin{gathered}
b_{x}=p_{x} a_{y}, \\
b_{y}=a_{y}+p_{y} a_{y}+p a_{y y}=\left(1+p_{y}\right) a_{y}+p a_{y y}, \\
b_{x x}=p_{x x} a_{y} .
\end{gathered}
$$

Then (1) and (2) imply

$$
a=b-p a_{y}=b-p / p_{x}\left(b_{x}\right),
$$

showing that $a$ is in $F\langle b\rangle$. (2) and (4) imply

$$
b_{x}-p_{x} b_{x x} / p_{x x}=0
$$

showing that $b$ satisfies a second order d.p. over $F$.

Equations (1), (2), and (3) may be solved for $a, a_{y}, a_{y y}$ in terms of $b, b_{x}$ and $b_{y}$. Hence, since $a, a_{y}, a_{y y}$ are algebraically independent over $F, b$ cannot satisfy a first order d.p. over $F$. Equation (6) yields the irreducible d.p. satisfied by $b$,

$$
A=u_{x}-p_{x} u_{x x} / p_{x x},
$$

which may be chosen as a d.p. in a characteristic set for $b$.

By Corollary 3 to Theorem $2, L_{1}(F ; a)=2$. Since $F\langle a\rangle=F\langle b\rangle$, $L_{1}(F ; b)=2$. By the same corollary, $A$ cannot be the sole d.p. in a characteristic set for $b$. 
14. A simply generated extension with no generator having a characteristic set of length one. Example: Let $P$ be the prime differential ideal generated by $u_{x}$ and $u_{y}$ over $F$. Then $u_{x}$ and $u_{y}$ also constitute a characteristic set of $P$. Let $a$ be a generic zero of $P$, and let $G=F\langle a\rangle$.

Then $h(F, n ; a)=1$, for all positive integral $n$. Hence $L_{1}(G / F)=0$. By Corollary 3 to Theorem 2, if $G$ has a generator $b$ with a characteristic set of length one, then $L_{1}(G / F)=m g$ where $g$ is the order of such characteristic set. Hence, $g=0$ and $b$ is algebraic over $F$. This implies that $a$ is algebraic over $F$ which is a contradiction of the fact that $a$ is a generic zero of $P$.

15. Specializations, $a^{\prime}$ is called a specialization of $a$ if there exists a differential homomorphism of $F\{a\}$ onto $F\left\{a^{\prime}\right\}$, taking $a$ into $a^{\prime}$, and leaving $F$ elementwise fixed. Since

$$
h\left(F, n ; a^{\prime}\right) \leqq h(F, n ; a), L\left(F ; a^{\prime}\right) \leqq L(F ; a) .
$$

We investigate when equality holds.

Proposition 1. If $a$ has a characteristic set of length one over $F$ and if $a^{\prime}$ is a proper specialization of $a$, (i.e., $F\{a\}$ and $F\left\{a^{\prime}\right\}$ are not isomorphic), then $L\left(F ; a^{\prime}\right)<L(F ; a)$.

Proof. Assume $L\left(F ; a^{\prime}\right)=L(F ; a)$, and let $A$ be a characteristic set for $a$ over $F$. Then by Corollaries 3 and 6 of Theorem 2, $A$ is also a characteristic set for $\alpha^{\prime}$ over $F$. Hence, $F\{a\}$ and $F\left\{a^{\prime}\right\}$ are isomorphic. Thus the proposition is proved.

Proposition 2. If $a$ has a characteristic set of length exceeding one, a proper specialization need not reduce the limit vector.

Proof. The following example will prove the point. As in the example of $\S 13$, we consider $F\{u\}$ with $u$ a differential indeterminate and two derivations denoted by subscripts $x$ and $y$. Let $t$ be a generic zero of the prime differential ideal $P$ in $F\{u\}$ with characteristic set and generator $u_{x}$. Also, let $a$ be a generic zero of the prime differential ideal $Q$ in $F\{u\}$ generated by and with a characteristic set, $u_{x x}$ and $u_{x y}$. Since $Q$ is properly contained in $P, t$ is a proper specialization of $a$. But, by direct computation, or by Corollary 4 of Theorem 2, $L(F ; a)=L(F ; t)=(0,2, \infty)$, proving the proposition.

16. Order of a prime ideal and systems of linear homogeneous d.p. Let $P$ be a prime differential ideal in $F\{y\}$ with generic zero $a$. 
The algebraic degree of transcendence of $F\langle a\rangle$ over $F$ is called the order of $P$, (ord $P)$. By Theorem 1, this is the number of parametric derivatives of $a$. We will show that this use of order agrees with the "order" of a system of linear homogeneous d.p. as used in the study of differential equations.

The following lemmas will lead to this result. The first two are nondifferential and are stated without proof. Lemma 3 is Kolchin's Lemma 1 in [4], where it is proved. Lemmas 4 and 5 are the differential analogues of Lemmas 1 and 2, respectively.

LEMMA 1. Let $S$ be a system of linear homogeneous polynomials in $K\left[x_{1}, \cdots, x_{n}\right]$ which is a vector space over $K$, where $K$ is a nondifferential field. Then

(a) (S) is prime and contains no linear homogeneous polynomials which are not in $S$.

$$
S \cap K\left[x_{1}, \cdots, x_{r}\right]=0=(S) \cap K\left[x_{1}, \cdots, x_{r}\right]=0 .
$$

LEMMA 2. Let a set $T$ of linear homogeneous polynomials with coefficients in a field $K$ generate a vector space over $K$ and over an extension $L$ of $K$. Then elements of $T$ linearly independent over $K$ remain linearly independent over $L$, and the number of such elements in a maximal set is the dimension of both vector spaces.

LEMMA 3. Let $C$ be the field of constants of the partial differential field $F$, and let $a_{1}, \cdots, a_{n}$ belong to $F$. If $a_{1}, \cdots, a_{n}$ are linearly dependent over $C$, then $W_{D_{1}, \cdots, D_{n}}\left(a_{1}, \cdots, a_{n}\right)=0$ for every choice of derivatives $D_{1}, \cdots, D_{n}$ where $W_{D_{1}, \cdots, D_{n}}\left(a_{1}, \cdots, a_{n}\right)=\operatorname{det}\left(D_{i} a_{j}\right)$. Conversely, if $W_{D_{1}, \cdots, D_{n}}\left(a_{1}, \cdots, a_{n}\right)=0$ for every choice of $D_{1}, \cdots, D_{n}$ of orders $\leqq n-1$, then $a_{1}, \cdots, a_{n}$ are linearly dependent over $C$.

LEMma 4. Let $S$ be a system of homogeneous linear partial d.p. in $F\{y\}$ which is a vector space over $F$ and is closed under the derivations $d_{1}, \cdots, d_{m}$. Let $T$ denote a set of $y$-derivatives. Then [S] is prime and

$$
S \cap F[T]=0 \Rightarrow[S] \cap F[T]=0 .
$$

Proof. Let $V$ denote the set of finite sets of $y$-derivatives. Then by Part (a) of Lemma 1, if $U \in V, S \cap F[U]$ generates a prime ideal $S_{U}$ in $F[U]$. Since the union of the $S_{\sigma}$ over all $U$ in $V$ is $[S],[S]$ is prime.

Let $T^{\prime}$ be a finite subset of $T$. By Part (b) of Lemma 1, 


$$
S \cap F\left[T^{\prime}\right]=0 \Rightarrow S_{T^{\prime}} \cap F\left[T^{\prime}\right]=0 \Rightarrow[S] \cap F[T]=0 .
$$

Lemma 5. Let $S$ denote a set of homogeneous linear partial d.p. in $F\{y\}$, and let $G$ be an extension of $F$. Let $T$ denote a set of $y$ derivatives. If $P$ and $Q$ denote the prime differential ideals generated by $S$ in

$$
F\{y\} \quad \text { and } \quad G\{y\} \quad \text { respectively, }
$$

then

$$
P \cap F[T]=0 \Leftrightarrow Q \cap G[T]=0 .
$$

This implies that $P$ and $Q$ have the same orders.

Proof. Let $S^{*}$ denote the system consisting of the d.p. in $S$ and of their derivatives. Let $B$ be a maximal linearly independent subset of $S^{*}$ over $F$, therefore, by Lemma 2 , over $G$. By Lemma $4, P$ contains a nonzero polynomial in members of $T$ if and only if it contains such a polynomial which is linear homogeneous; that is, if and only if there is a linear dependence among members of $T$ and $B$ over $F$. In the same way $Q$ contains a nonzero polynomial in the members of $T$ if and only if the members of $T$ and $B$ are linearly dependent over $G$. But Lemma 2 shows that these conditions are equivalent. Thus the lemma is proved.

THEOREM 7. Let $S$ be a system of homogeneous linear partial d.p. in $F\{y\}$. If the set of solutions of $S$ is of linear dimension $k$ over constants, then ord $[S]=k$.

Proof. (a) ord $[S] \geqq k$ :

Let $u_{1}, \cdots, u_{k}$ be a linear basis for the solutions over constants. Let $c_{1}, \cdots, c_{k}$ be new constants algebraically independent over $F\left\langle u_{1}, \cdots, u_{k}\right\rangle$. Let $v=\sum c_{i} u_{i}$. Since the $u_{i}$ are linearly independent over constants, by Lemma 3 , for some set of derivatives $D_{1}, \cdots, D_{k}$ of orders $\leqq k-1$, $W_{D_{1}}, \cdots,{ }_{D_{k}}\left(u_{1}, \cdots, u_{k}\right) \neq 0$. Hence, $D_{1} v, \cdots, D_{k} v$ are linearly independent over $F$. Since $v$ belongs to the manifold of $[S],[S]$ contains no linear homogeneous polynomial, and therefore by Lemma 4, contains no polynomial in $D_{1} y, \cdots, D_{k} y$. Hence, ord $[S] \geqq k$.

$$
\text { (b) } k \geqq \operatorname{ord}[S] \text { : }
$$

Let $\operatorname{ord}[S]=k$. Let $v_{1}$ be a generic zero of $[S]$. Then $k$ derivatives, $D_{1}, \cdots, D_{k}$, of $v_{1}$ are algebraically independent over $F$. We define inductively a sequence of elements $v_{j}$ which are solutions of $S$ by the requirement that $v_{j}$ be a generic zero of the differential ideal generated 
by $S$ over $F\left\langle v_{1}, \cdots, v_{j-1}\right\rangle\{y\}$.

Then, by Lemma $5, D_{1} v_{j}, \cdots, D_{k} v_{j}$ are algebraically independent over $F\left\langle v_{1}, \cdots, v_{j-1}\right\rangle$. In particular, the $D_{i} v_{j}, i, j=1, \cdots, k$, are algebraically independent over $F$. Hence, $W_{D_{1}, \cdots, D_{k}}\left(v_{1}, \cdots, v_{k}\right) \neq 0$. Then Lemma 3 implies that the $v_{j}$ are linearly independent over constants. Thus $k \geqq \operatorname{ord}[S]$.

Theorem 8. Let $P$ be a prime differential ideal in $F\{y\}$. If $P$ contains a set of nonzero d.p. $A_{i}, i=1, \cdots, m$, such that $A_{i}$ involves only $y$-derivatives of the form $d_{i}^{t} y$ where $t$ is a nonnegative integer, then ord $P$ is finite.

Proof. If $P$ contains a d.p. free of proper $y$-derivatives, then ord $P=0$, and the theorem holds. Hence, we may assume that the $A_{i}$ involve proper $y$-derivatives. Then $A_{i}$ involves a derivative of the leader of some member of a characteristic set $C$ of $P$. Hence, there exist leaders of members of $C$ of the form $d_{i}^{t_{i}} y, t_{i}>0, i=1, \cdots, m$. Thus, by Theorem 1 , ord $P \leqq t_{1} \cdots t_{m}$. proving the theorem.

If we specify in Theorem 8 that $P=[S]$, where $S$ is a system of linear partial homogeneous d.p. in $F\{y\}$, then we have the hypothesis of a theorem of Delsarte [1], Proposition A, page 37. Then by Theorems 7 and 8, the linear dimension of the solution space of $S$ is finite, which is Delsarte's conclusion.

\section{REFERENCES}

1. Jean Delsarte, Sur certains systèmes d'équations aux dérivées partielles à une seule fonction inconnue, et sur une généralization de la théorie des fonctions de Bessel et des fonctions hypergéométriques, Premier Colloque Sur Les Équations aux Dérivées Partielles held at Louvain, December 17-19, 1962, pp. 35-62.

2. E.R. Kolchin, Extensions of differential fields, II, Ann. of Math. 45 (1945), 358-361. 3. - Extensions of differential fields, III, Bull. Amer. Math. Soc. 53 (1947), 399-401.

4. , Picard-Vessiot theory of partial differential fields, Proc. Amer. Math. Soc. 3 (1952), 596-603.

5. H.W. Raudenbush, Hypertranscendental adjunctions to partial differential fields, Bull. Amer. Math. Soc. 40 (1934), 714-720.

6. J.F. Ritt, Differential algebra, Amer. Math. Soc. Colloquium Publications, vol. 33, New York, 1950.

7. B.L. Van Der Waerden, Modern algebra, Volume 1, Revised English Edition, F. Ungar Publishing Co., New York, 1953, Section 33, pp. 99-104.

VASSAR COLLEgE 



\section{PACIFIC JOURNAL OF MATHEMATICS}

\section{EDITORS}

H. Samelson

Stanford University

Stanford, California

R. M. Blumenthal

University of Washington

Seattle, Washington 98105
J. Dugundu

University of Southern California Los Angeles, California 90007

*Richard Arens

University of California

Los Angeles, California 90024

\section{ASSOCIATE EDITORS}
E. F. BECKENBACH
B. H. NeumanN
F. WOLF
K. YOSIDA

\section{SUPPORTING INSTITUTIONS}

UNIVERSITY OF BRITISH COLUMBIA CALIFORNIA INSTITUTE OF TECHNOLOGY UNIVERSITY OF CALIFORNIA MONTANA STATE UNIVERSITY

UNIVERSITY OF NEVADA

NEW MEXICO STATE UNIVERSITY

OREGON STATE UNIVERSITY

UNIVERSITY OF OREGON

OSAKA UNIVERSITY

UNIVERSITY OF SOUTHERN CALIFORNIA
STANFORD UNIVERSITY

UNIVERSITY OF TOKYO

UNIVERSITY OF UTAH

WASHINGTON STATE UNIVERSITY

UNIVERSITY OF WASHINGTON

AMERICAN MATHEMATICAL SOCIETY CALIFORNIA RESEARCH CORPORATION SPACE TECHNOLOGY LABORATORIES NAVAL ORDNANCE TEST STATION 


\section{Pacific Journal of Mathematics}

\section{Vol. 15, No. $1 \quad$ September, 1965}

Donald Charles Benson, Unimodular solutions of infinite systems of linear

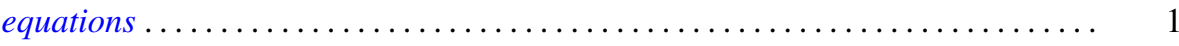

Richard Earl Block, Transitive groups of collineations on certain designs . . . . . . 13

Barry William Boehm, Existence of best rational Tchebycheff approximations .... . 19

Joseph Patrick Brannen, A note on Hausdorff's summation methods . . . . . . . . . . 29

Dennison Robert Brown, Topological semilattices on the two-cell ............ 35

Peter Southcott Bullen, Some inequalities for symmetric means . . . . . . . . . . 47

David Geoffrey Cantor, On arithmetic properties of coefficients of rational

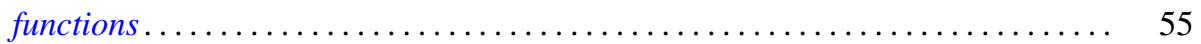

Luther Elic Claborn, Dedekind domains and rings of quotients . . . . . . . . . 59

Allan Clark, Homotopy commutativity and the Moore spectral sequence ........ 65

Allen Devinatz, The asymptotic nature of the solutions of certain linear systems of

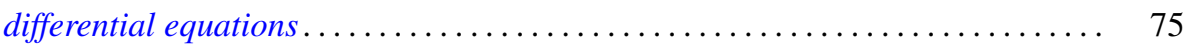

Robert E. Edwards, Approximation by convolutions ................... 85

Theodore William Gamelin, Decomposition theorems for Fredholm operators . . . . . 97

Edmond E. Granirer, On the invariant mean on topological semigroups and on

topological groups .................................. 107

Noel Justin Hicks, Closed vector fields . . . . . . . . . . . . . . . . . . . 141

Charles Ray Hobby and Ronald Pyke, Doubly stochastic operators obtained from

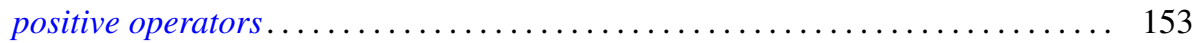

Robert Franklin Jolly, Concerning periodic subadditive functions . . . . . . . . . 159

Tosio Kato, Wave operators and unitary equivalence . . . . . . . . . . . . . . 171

Paul Katz and Ernst Gabor Straus, Infinite sums in algebraic structures . . . . . . . 181

Herbert Frederick Kreimer, Jr., On an extension of the Picard-Vessiot theory ...... 191

Radha Govinda Laha and Eugene Lukacs, On a linear form whose distribution is

identical with that of a monomial ......................... 207

Donald A. Ludwig, Singularities of superpositions of distributions . . . . . . . . . 215

Albert W. Marshall and Ingram Olkin, Norms and inequalities for condition

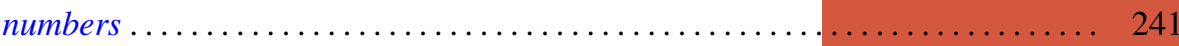

Horace Yomishi Mochizuki, Finitistic global dimension for rings . . . . . . . . . . 249

Robert Harvey Oehmke and Reuben Sandler, The collineation groups of division

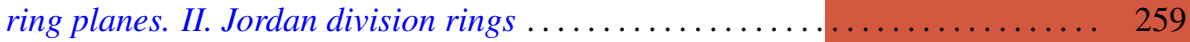

George H. Orland, On non-convex polyhedral surfaces in $E^{3} \ldots \ldots \ldots \ldots \ldots \ldots \ldots 267$

Theodore G. Ostrom, Collineation groups of semi-translation planes . . . . . . . . 273

Arthur Argyle Sagle, On anti-commutative algebras and general Lie triple

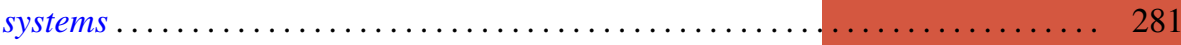

Laurent Siebenmann, A characterization of free projective planes . . . . . . . . . 293

Edward Silverman, Simple areas.................................. 299

James McLean Sloss, Chebyshev approximation to zero .................. 305

Robert S. Strichartz, Isometric isomorphisms of measure algebras . . . . . . . . . 315

Richard Joseph Turyn, Character sums and difference sets . . . . . . . . . . . . 319

L. E. Ward, Concerning Koch's theorem on the existence of arcs . . . . . . . . . . 347

Israel Zuckerman, A new measure of a partial differential field extension ......... 357 